\title{
»Providence leaves no real room to fortuna«: Vom Zufall bei Augustinus
}

\author{
Christoph Markschies
}

Mein Titel dürfte nicht überraschen, um es vorsichtig zu formulieren. Obwohl er aus einem einschlägigen Lexikonartikel zitiert ist, ${ }^{1}$ bedient er Vorurteile. Schließlich gilt der nordafrikanische Bischof Augustinus von Hippo allgemein - da muss man ihn gar nicht so sehen wie Kurt Flasch - mindestens in seinen späteren Jahren als ein klassischer Vertreter einer strengen Prädestinationslehre, die per definitionem keinen wirklichen Raum für den Zufall lässt, jedenfalls dann, wenn man diesen verwirrend unpräzisen Terminus als Konkurrenzbegriff zu »Schicksal« und »Vorsehung« verwendet, wie der entsprechende Artikel im »Historischen Wörterbuch der Philosophie« als eine mögliche Bedeutungsdimension anbietet. ${ }^{2} »$ Providence leaves no real room to $f[$ ortuna]«, lautet entsprechend auch eine Überschrift in dem knappen Artikel, den Jan den Boeft zum Lemma fortuna im renomierten »Augustinus-Lexikon" geschrieben hat (und man muss sich klarmachen, dass gut ein Drittel der knapp

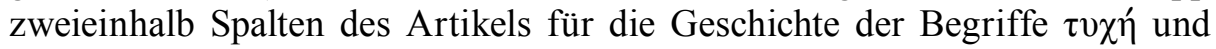
fortuna vor Augustinus verwendet wird und der Autor dann konstatiert: »In Augustin's œuvre $f$ [ortuna] is not an important theme «). ${ }^{3}$ Allerdings steht im erwähnten Artikel im »Historischen Wörterbuch der Philosophie« eben auch zu lesen, dass Termini wie eben $\tau$ $\chi \eta$ und fortuna, »die vielfach als Vorläuferausdrücke« unseres Begriffs Zufall gelten, »bei präziser Übersetzung eher nicht mit $\backslash Z[$ ufall] $<$ wiederzugeben« sind, als vielmehr »die notwendige oder glückliche $\gg$ Fügung « «, mit einer $» B e d e u t u n g s k o m p o n e n t e ~ v o n ~ \gg$ Schicksal $\ll{ }^{4}{ }^{4}$ Übrigens hält der Artikel auch fest, dass die Übersetzung von contingentia mit dem Begriff »Zufälligkeit« etabliert, aber irreführend sei, ${ }^{5}$ so dass man sich als schlichter Leser fragt, ob Zufall in dem schlichten Sinn, »dass es für ein Ereignis keine Ursachen gibt oder dass solche jetzt nicht oder prinzipiell nicht erkennbar sind«, dann in der Antike überhaupt auf den Begriff gebracht

\footnotetext{
Den Boeft (2004), 57.

Kranz (2004), 1408.

Den Boeft, $56 \mathrm{f}$.

Kranz (2004), 1409.

5 Kranz (2004), 1409.
} 
werden konnte. ${ }^{6}$ Für Augustinus stellt sich die Frage noch einmal radikaler: Weder die schöne barocke Concordantia Augustiana aus dem Jahre $1656^{7}$ noch das erwähnte Augustinus-Lexikon vom Jahr 1994 bieten überhaupt ein Lemma contingentia. Und auch die Ausbeute des im »Corpus Augustinianum Gissense« voll digitalisierten Euvres ist mehr als mager, ein einziger und für unsere Zusammenhänge nicht einschlägiger Beleg. ${ }^{8}$ Mit anderen Worten: contingentia verwendet Augustinus als Terminus offenbar nicht und auch der Begriff fortuna spielt keine große Rolle bei ihm.

Nun soll es auf dieser Jahrestagung freilich um »Transformationen der contingentia selbst, ... in ihrer Verbildlichung (z. B. als Tyche, Fortuna etc.), aber auch ihrer historischen Semantik .... und insbesondere um die Transformation antiker Modelle der Kontingenzbewältigung gehen. Damit wird der nordafrikanische Bischof Augustinus aber wieder höchst einschlägig für einen Beitrag. Man muss nicht wie Hermann Lübbe alle Religion als »Kontingenzbewältigungspraxis« deuten (und Theologie dann also als die rationale Reflexion solcher Kontingenzbewältigungspraxis), um sofort an Augustinus und seine erwähnten Ansichten über die Prädestination als ein besonders einflussreiches antikes Modell der Kontingenzbewältigung zu denken. Schließlich gilt, wie Hans Freiherr von Campenhausen einmal bemerkt hat: »Augustin ist der einzige Kirchenvater, der bis auf diesen Tag eine geistige Macht geblieben ist «. ${ }^{9}$

Genug der Vorrede. Beginnen wir also Augustinus unter der Frage darzustellen, welche Modelle der Kontingenzbewältigung sich bei ihm finden. Was ist »Kontingenzbewältigung «? Niklas Luhmann versteht »kontingent« als etwas, "was weder notwendig ist noch unmöglich ist; was also so, wie es ist (war, sein wird), sein kann, aber auch anders möglich ist. Der Begriff bezeichnet mithin Gegebenes (zu Erfahrendes, Erwartetes, Gedachtes, Phantasiertes) im Hinblick auf mögliches Anderssein; er bezeichnet Gegenstände im Horizont möglicher Abwandlungen «. ${ }^{10}$ Kontingenzbewältigung wäre also jeder bewältigende Umgang mit der irritierenden Erfahrung, dass etwas im Leben und sogar das ganze Leben als weder notwendig noch unmöglich empfunden wird, als etwas, das »also so, wie es ist (war, sein wird), sein kann, aber auch anders möglich ist«. Genau aber dieses Problem beschäftigt Augustinus bereits in seiner frühesten erhaltenen Schrift, dem Dialog Contra Academicos. Der Autor hat seine Schrift selbst datiert auf die Tage zwischen den Semesterferien im Mailänder Residenzstadt-Rhetoren-Amt im August 386 n. Chr. und seiner

\footnotetext{
$6 \quad$ Kranz (2004), 1408.

7 Lenfant (1656), s.v.

8 Augustinus, div. quaest. 69,2 (CChr.SL 44 A, 186,49-51 Mutzenbecher): solet circumstantia scripturae inluminare sententiam, cum ea quae circa scripta sunt praesentem quaestionem contingentia diligenti discussione tractantur.

9 Freiherr von Campenhausen (1995), 151.

10 Luhmann (2010), 152; vgl. Lübbe (1998), 35-47.
} 
Taufe durch den Ortsbischof Ambrosius in der Osternacht im April 387 n. Chr.; der sich in einem innerlichen Transformationsprozess befindliche Rhetor hielt sich damals bekanntlich auf dem rund vierzig Kilometer nordwestlich von Mailand gelegenen Landgut Cassiciacum mit einer Reihe von Freunden wie Weggefährten auf. ${ }^{11}$ Der ganze Text ist, ausweislich seines an einen nordafrikanischen Freund namens Romanianus ${ }^{12}$ gerichteten Beginnes, ein Versuch der Kontingenzbewältigung:

Könnte doch die Tugend, Romanianus, einen Menschen, der für sie angelegt ist, dem widerstrebenden Schicksal ebenso entreißen, wie sie sich umgekehrt von niemandem entreißen läßt! Dann hätte sie bestimmt schon ihre Hand auf dich gelegt und dich für ihr rechtmäßiges Eigentum erklärt, hätte dich in den Besitz wirklich sicherer Güter hinüber geleitet und dich nicht einmal günstigen Zufällen unterworfen sein lassen. ${ }^{13}$

Virtus, unvollkommen als »Tugend« übersetzt, ist, wie Karin Schlapbach in ihrem gründlichen Kommentar gezeigt hat, »sowohl machtlos wie allmächtig gegen fortuna«, die hier als »Schicksal « wiedergegeben ist. ${ }^{14}$ Machtlos ist die virtus beim Toren (stultus), der ohne Tugend lebt, allmächtig ist sie beim sapiens, von dem die klassische, bei Diogenes Laertius, Cicero, Philo und Clemens

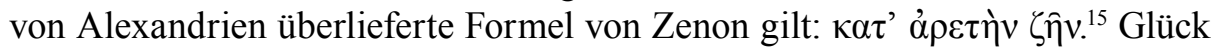
bedeutet in diesem Zusammenhang Besitz der Tugend und Unabhängigkeit von äußeren Gütern. ${ }^{16}$ Fortuna ist einerseits ein Widerpart von virtus, andererseits führt sie zur Weisheit und kann - wie wir gleich sehen werden - von Augustinus sogar mit providentia gleichgesetzt werden und erweist sich daher als notwendige Voraussetzung zur Erlangung des Glücks. Wer in einem quasi juristischen Sinne »rechtmäßiges Eigentum« der virtus geworden ist, also in einem unauflöslichen Prägungsverhältnis zu ihr steht, im Besitz »wirklich sicherer«, d. h. unverlierbarer »Güter« (vor allem der vier Kardinaltugenden) ${ }^{17}$, der wird kein Sklave selbst günstiger (und erst recht ungünstiger) Zufälle: ne prosperis quidem casibus servire. »Zufall« übersetzt hier also casus, man könnte vielleicht »Umstand « oder »Vorkommnis« sagen, etwas, was im Sinne der zitierten Definition Luhmanns $» s o$, wie es ist (war, sein wird), sein kann, aber auch an-

11 Augustinus, Conf., IX 4,7 (CChr.SL 27, 136,1-19 Verheijen) sowie Augustinus, Retract., I 1,1 (CChr.SL 57, 7,1-11 Mutzenbecher) mit Fuhrer (1997), 3.

12 Fuhrer (1997), $4 \mathrm{f}$.

13 Augustinus, Acad., I 1 (CChr.SL 29, 3,1-6 Green): O utinam, Romaniane, hominem sibi aptum ita uicissim uirtus fortunae repugnanti posset auferre, ut ab ea sibi auferri neminem patitur, iam tibi profecto iniecisset manus, suique iuris te esse proclamans, et in bonorum certissimorum possessionem traducens ne prosperis quidem casibus seruire permitteret. - Die Übersetzung entnehme ich: Augustinus (1987), 44.

14 K. Schlapbach (2003), 28.

15 SVF 1, nr. $179 \mathrm{f} ., 45 \mathrm{f}$.

16 SVF 3, nr. 49-67, 13-16.

17 Schlapbach (2003), 30. 
ders möglich ist«. Augustinus interessiert in diesen Jahren offenkundig nicht die bei Luhmann im Hintergrund stehende Frage kausaler Determination, sondern die Frage, ob man »ein Sklave« der so oder so möglichen Vorkommnisse ist oder eben nicht, mithin eine Frage der inneren Haltung oder Einstellung gegenüber den Vorkommnissen. Bemerkenswerterweise identifiziert Augustinus schon in diesem frühen Text, wie wir bereits sagten, fortuna mit providentia:

Doch ist es nun einmal so eingerichtet - ob entsprechend unseren Verdiensten oder mit Naturnotwendigkeit -, dass den göttlichen Geist im sterblichen Wesen, wenn er Vergänglichem anhängt, die Weisheit durchaus nicht in ihren Hafen aufnimmt, wo er weder vom widrigen noch vom günstigen Wind des Schicksals bewegt würde - es sei denn, das Schicksal selber, freundlich oder scheinbar feindlich, führe ihn dorthin. Infolgedessen bleiben uns für dich nur Gebete zu jenem Gott, der diesen Dingen seine Sorge zuwendet, Gebete, in denen wir, wenn möglich, erwirken möchten, dass er dich dir zurückgibt (...) und deinen Geist, der sich seit langem schon wieder frei zu atmen sehnt, am Ende in die Luft wahrer Freiheit aufsteigen lässt. ${ }^{18}$

Auch wenn diese Passage schon sehr klassisch christlich klingt, von merita und necessitas naturae die Rede ist, geht es nicht um die Geschichte vom Sündenfall, der dadurch entstandenen Natur und den mit solcher Natur erworbenen oder eben nicht erworbenen Verdiensten - erst in seinen späten Retractationes von 426/427 n. Chr. möchte Augustinus diese Stelle so gelesen wissen. ${ }^{19}$ Hier geht es um das neuplatonische Motiv der Wahl, die die präexistente Seele im Blick auf ihre Verbindung mit der Materie trifft, und ein gegenteiliges eher deterministisches Modell, das man beispielsweise bei Cicero findet ${ }^{20}$ - in jenem Dialog Hortensius, der den neunzehnjährigen Augustinus begeisterte (also rund dreizehn Jahre vor Abfassung der Dialoge Contra Academicos) und in einem freilich schwer zu bestimmenden Verhältnis zu unserem Frühdialog steht. ${ }^{21}$ Aus der selbstgewählten Fremde ist Rückkehr in den Hafen der Philosophie, in die eigentliche Heimat (wie in ganz traditioneller, im Neuplatonismus gern aufgegriffener Metaphorik formuliert wird) nur möglich, wenn fortuna ganz providentiell die Menschen zur virtus hin geleitet, als ein Gnadengeschenk, für das man freilich beten kann. Augustinus kann daher sagen:

18 Augustinus, Acad., I 1 (CChr.SL 29, 3,6-15 Green): Sed quoniam ita comparatum est siue pro meritis nostris siue pro necessitate naturae, ut diuinum animum mortalibus inhaerentem nequaquam sapientiae portus accipiat, ubi neque aduersante fortunae flatu neque secundante moueatur, nisi eo illum ipsa uel secunda uel quasi aduersa perducat, nihil pro te nobis aliud quam uota restant, quibus ab illo cui haec curae sunt deo, si possumus, impetremus, ut te tibi reddat - ita enim facile reddet et nobis - sinatque mentem illam tuam, quae respirationem iam diu parturit, aliquando in auras uerae libertatis emergere.

19 Augustinus, Retract., I 1,2 (CChr.SL 57, 7,12-23 Mutzenbecher).

20 Schlapbach (2003), 32: Cicero, Hort., fr. 110 Grilli = 101 Straume-Zimmermann.

21 Schlapbach (2003), 13-25. 
Vielleicht unterliegt nämlich, was man allgemein Schicksal nennt, auch einer verborgenen Ordnung, nennen wir doch in den Ereignissen nichts anderes Zufall als das, dessen Grund und Ursache uns verschlossen ist; und nichts Günstiges oder Ungünstiges dürfte dem Einzelnen widerfahren, das nicht zum Ganzen passte und zu ihm stimmte. Diesen Gedanken, durch Offenbarungssprüche voll fruchtbarster Lehren verkündet, weit entfernt von der Denkweise der Uneingeweihten, verspricht die Philosophie, für die ich dich gewinnen möchte, ihren wahren Liebhabern zu zeigen. ${ }^{22}$

Auch wenn diese Erklärung von fortuna, Schicksal, tentativ gegeben wird (etenim fortasse), bezieht sich Augustinus auf längst etablierte philosophische Konsense stoischer und neuplatonischer Provenienz. Schicksal, fortuna, und casus, Zufall, sind Ausdruck verborgener Ordnung und nur noch nicht erkannter Ursachen. Dieses spezifische, alltagsdistante philosophische Verständnis von Schicksal, fortuna, und casus, Zufall, ist entweder autoritativ durch oracula zu gewinnen oder durch rationalen Aufweis - es spricht viel dafür, mit Karin Schlapbach anzunehmen, dass hier autoritative philosophische Wissensquellen, beispielsweise auch die von Porphyrius kommentierten oracula gemeint sind, die offenbar längst vorliegen und interpretiert werden können, und jedenfalls noch nicht die christlichen autoritativen Offenbarungstexte. ${ }^{23}$

Im weiteren Verlauf des Dialoges, der nach einer durchaus nicht unwahrscheinlichen historischen Stilisierung sieben Tage später als der eben behandelte Beginn gehalten wurde, äußert sich Alyphius, ein familiarissimus amicus des Augustinus, Finanzbeamter und späterer Bischof von Thagaste ${ }^{24}$ wie folgt:

Welchen Einflussbereich du dem Schicksal zuweist, sehe ich noch nicht recht ein. Wenn du nämlich meinst, um das Schicksal verachten zu können, sei man auf es selbst angewiesen, dann schließe auch ich mich dir als Begleiter in dieser Auffassung an. Wenn du dem Schicksal aber keinen anderen Einfluss zubilligst als den, dass nur mit seinem Willen die Bedürfnisse des Lebens befriedigt werden, können, dann bin ich anderer Ansicht. Denn entweder ist es möglich, dass der noch nicht Weise, aber nach der Weisheit Verlangende gegen den Willen und Widerstand des Schicksals sich das nimmt, was wir als für das Leben erforderlich ansehen, oder man muss zugeben, dass es auch im ganzen Leben des Weisen eine beherrschende Stellung einnimmt, da auch der Weise selbst auf das, was für den Leib vonnöten ist, nicht verzichten kann. ${ }^{25}$

22 Augustinus, Acad., I 1 (CChr.SL 29, 3,15-22 Green): Etenim fortasse quae uulgo fortuna nominator occulto quodam ordine regitur nihilque aliud in rebus casum uocamus, nisi cuius ratio et causa secreta est, nihilque seu commodi seu incommodi contingit in parte, quod non conueniat et congruat uniuerso. Quam sententiam uberrimarum doctrinarum oraculis editam remotamque longissime ab intellectu profanorum se demonstraturam ueris amatoribus suis ad quam te inuito philosophia pollicetur.

23 Schlapbach (2003), 39.

24 Augustinus, Acad., III 6,13 (CChr.SL 29, 42,1-24 Green).

25 Augustinus, Acad., III 2,2 (CChr.SL 29, 35,8-18 Green): Quantum iuris, inquit, fortunae tribuas, nondum bene noui. Nam si ad contemnendam fortunam fortuna ipsa opus esse arbitraris, me quoque comitem in hanc sententiam do tibi. Sin fortunae nihil aliud concedis quam ea, quae 
Therese Fuhrer hat diese Passage in ihrem feinen Kommentar einem »Binnenproömium « zugewiesen, in dem es um die Bedeutung der fortuna für das sapientiae studium geht. ${ }^{26}$ Es geht also nochmals um die schon zu Beginn des ersten Buches angeschnittene Frage, ob der, der die Weisheit studiert, beim Weg zur Weisheit (oder metaphorisch gesprochen: in den Hafen und die Heimat zurück) von der Gunst der fortuna abhängig ist. Alyphius und sein Freund Augustinus differieren in ihren Positionen. Für Augustinus gilt - wie schon am Beginn des ersten Buches - fortuna als, wie Augustinus hier sagt, divinum auxilium, als unentbehrliches göttliches Hilfsmittel. ${ }^{27}$ Das Proömium zeigt (natürlich auch aus rein topischen Gründen), dass der Autor zu solchem günstigen Schicksal übrigens auch die günstigen materiellen Umstände in Cassiciacum zählt, also die Tatsache, dass man von Geldsorgen frei über fortuna und casus nachdenken kann. Allerdings erfährt man hier im Fortgang, dass dieses göttliche Hilfsmittel fortuna für Augustinus nur den Weg zur Philosophie bahnt und danach entbehrlich wird, ja sogar verachtet werden kann. Alyphius dagegen orientiert sich an der konventionellen Vorstellung der fortuna als einer unberechenbaren $\tau \dot{\chi} \chi \eta$, die es zu bezwingen gilt. ${ }^{28}$ Das Bild des Augustinus ist ungleich positiver; wenn der, der die Weisheit sucht, einmal zum Weisen und Philosophen geworden ist (von ihm gilt: sapientiam scire und scientia sapientiae), ${ }^{29}$ braucht er fortuna nicht mehr - auch das ist in einem ganz präzisen Sinne gemeint: Natürlich braucht er fortuna als Mensch, den bestimmte leibliche Bedürfnisse prägen; aber als Philosoph, der die Weisheit besitzt, braucht er keine Gunst des Schicksals mehr zu ihrer Erlangung. Man muss sich, wie Therese Fuhrer richtig bemerkt hat, den antiskeptischen Duktus der Argumentation klarmachen, wenn man sich wundert, wie in einer so stark neuplatonisch gefärbten Diskussionsrunde so schlicht vom Besitz der Weisheit als einem Wissen der Weisheit gesprochen werden kann: sapiens scit scientiam ist zwar eine tautologische Formulierung, aber zugleich die wohl stärkste antiskeptische Aussage, die man sich in unserem Zusammenhang denken kann.

Ich verfolge nun die Thematik von fortuna und casus nicht in den übrigen Frühdialogen des Augustinus, zu ähnlich ist der Befund in De ordine und De beata vita zu dem, was wir in Contra Academicos beobachtet haben. Mich interessiert vielmehr der spätere und späte Augustinus, um nach Transformationen der Kontingenzbewältigungspraktiken beim Bischof von Hippo Regius

corporis necessitati non possunt nisi ipsa uolente suppetere, non ita sentio. aut enim licet eadem repugnante atque inuita nondum sapienti, cupido tamen sapientiae ea sumere, quae uitae necessaria confitemur, aut concedendum est etiam in omni sapientis uita eam dominari, cum et ipse sapiens his, quae corpori necessaria sunt, non indigere non possit.

26 Fuhrer (1997), 233.

27 Augustinus, Acad., II 1,1 (CChr.SL 29, 18,19 Green).

28 Fuhrer (1997), 238.

29 Fuhrer (1997), 246. 
gegenüber dem Mailänder Stadtrhetor in beruflicher wie persönlicher Midlife-Crisis zu fragen.

Dazu muss man sich klarmachen, dass es gerade einmal zehn Jahre nach den Frühdialogen in Cassiciacum zu einer, wie mir jedenfalls scheint, doch radikalen Wende im Denken des Augustinus kommt, nämlich zur Zuspitzung der einschlägigen Gedanken des Augustinus in den Quaestiones ad Simplicianum von 396/397 n. Chr. aufgrund einer gründlichen Beschäftigung mit dem paulinischen Römerbrief. Diesen Text hat Kurt Flasch bekanntlich unter der Überschrift »Logik des Schreckens« ediert und die zugrundeliegende Anthropologie ebenso wie den radikalen Determinismus als »Nierenstein in den Nieren Alteuropas « und unerträgliche Verfinsterung einer angeblich lichten Antike gegeißelt. ${ }^{30}$ In einem vor einiger Zeit erschienenen Beitrag ging Flasch noch weiter und baut neben Augustinus als dem Verfinsterten und Dunkelmann Origenes als die helle Lichtgestalt der antiken christlichen Theologiegeschichte auf - ein doppelter Ausgang der christlichen Antike, dem nach Flasch ein doppelter Ausgang des Mittelalters im irenisch-freundlichen alter Origenes, Erasmus von Rotterdam, und im alter Augustinus, dem Augustinereremiten Martin Luther entspricht. ${ }^{31}$ Reine Dualismen, noch dazu kombiniert mit der Aufforderung, sich gefälligst für eine der beiden Optionen zu entscheiden, befriedigen natürlich wenig, selbst wenn sie äußerst unterhaltsam vorgetragen werden. Sie verlocken eher dazu, Augustinus noch einmal hervorzunehmen und nach den Gründen für die Transformationen seines Begriffs von fortuna und casus in späteren Lebensjahren zu fragen.

Die neue Gnadentheologie, die Augustinus als Frucht einer neuen Pauluslektüre ab 394/395 n. Chr. entwickelte und erstmals abschließend 396/397 n. Chr. in den Quaestiones ad Simplicianum formulierte, gipfelt in der Vorstellung von einer massa damnata, die - wie ich anderswo gezeigt habe ${ }^{32}-$ ganz deutlich gegen die Konzeption des Origenes von einer eschatologischen $\mathrm{Zu}-$ rechtbringung aller gerichtet ist, die wir mit einem Stichwort aus der Apostel-

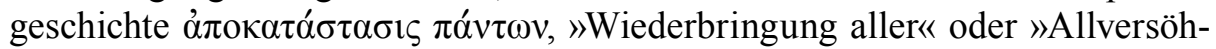
nung « nennen (obwohl Augustinus Origenes im Original gar nicht lesen konnte und Origenes die Allversöhnung auch nicht lehrt, sondern lediglich zu lehren erwägt). Wenn ich recht sehe, beginnt Augustinus in seiner Expositio quarundam propositionum ex epistula apostoli ad Romanos von 394 n. Chr., der ersten Schrift aus einer Reihe kleinerer Kommentare und Abhandlungen, die sich der neuen Pauluslektüre verdanken, ganz zaghaft im Blick auf die Menschheit von einer massa zu reden - alle Menschen, so sagt er, beginnen ihren Lebensweg gleich sündig, als ob sie aus demselben Lehmklumpen geformt wären, und sind insofern eine massa der Menschheit, Juden, Christen, Heiden, ohne

30 Flasch (1995).

31 Flasch (2009), 472-502.

32 Markschies (2012), 310-314. 
Unterschied, ohne Entschuldigung. ${ }^{33}$ Damit ist der Impetus der Kontingenzbewältigung natürlich radikal different von den hellen, optimistischen Tönen der Frühdialoge von Cassiciacum und es ist kein Zufall, dass in dieser und der folgenden Schrift an Simplicianus das Stichwort fortuna überhaupt nicht fällt.

In den zwei Jahren zwischen der Expositio quarundam propositionum ex epistula apostoli ad Romanos und den Quaestiones ad Simplicianum, also zwischen 394/395 und 396/397 n. Chr., hat Augustinus seine Interpretation des paulinischen Römerbriefs nochmals radikalisiert und wollte nun keinerlei Eigeninitiative des Menschen im Zusammenhang seiner Berufung, keine Verdienste, keine Willensregung mehr akzeptieren. Daher verschärfte sich auch der Wortgebrauch im Blick auf das Wort massa. In den Antworten auf die Fragen des Mailänder Presbyters Simplicianus liegt nunmehr eine konsequente Terminologie der massa damnata vor, nicht mehr vereinzelte und von biblischen Töpfergleichnissen motivierte Bezugnahmen wie noch zwei Jahre zuvor. Augustinus spricht von massa peccati, massa peccatorum et impiorum und entwirft das Bild einer umfassend geschädigten moralischen Autonomie des Menschen. ${ }^{34}$ In den Bekenntnissen hat Augustinus diese exegetischen Einsichten zum Römerbrief des Apostels Paulus dann an seiner eigenen Biographie expliziert, besser vielleicht: seine Biographie entsprechend seinen Einsichten konstruiert.

Entsprechend heftig polemisiert der gealterte Bischof in seinem großen Werk De civitate Dei um 415 n. Chr. gegen fortuna, wobei er unter diesem Begriff nun die pagane Göttin und keinen primär unschuldigen philosophischen Terminus mehr begreift. ${ }^{35}$

Wie kann denn die Göttin Fortuna bald gut, bald böse sein? Oder ist sie, wenn sie böse ist, keine Göttin mehr, sondern hat sich plötzlich in einen boshaften Dämon verwandelt? Aber wie viele solche Göttinnen gibt es eigentlich? Doch wohl, sollte man meinen, ebenso viel gute Geschicke wie vom guten Geschick erfreute Menschen. Und da es zugleich zur selben Zeit auch eine Menge Menschen gibt, die vom bösen Geschick betroffen sind, wäre dann nicht Fortuna, wenn es immer nur eine wäre, zugleich gut und böse, gut für die einen und böse für die anderen? Oder ist die Fortuna, die Göttin ist, immer gut? ... Wo aber bleibt dann die Begriffsbestimmung der Fortuna? Und was soll's, dass sie vom zufälligen Geschick sogar ihren Namen bekommen hat? Ist sie zufälliges Geschick, kann ihre Verehrung ja

33 Augustinus, Exp. prop. Rom. 54,18f (CSEL 84, 38,22-39,6 Divjak): O homo, tu quis es, qui respondeas deo? Numquid dicit figmentum ei, qui se finxit: quare sic me fecisti? Annon habet potestatem figulus luti ex eadem conspersione vas facere, aliud quidem in honorem, aliud in contumeliam? (19) Quamdiu figmentum es, inquit, et ad massam luti pertines nondum perductus ad spiritualia, ut sis spiritualis omnia iudicans et a nemine iudiceris, cohibeas te oportet ab huiusmodi inquisitione et non respondeas deo.

34 Frederiksen (2007), 292.

35 Vgl. Bardy (1959), $810 \mathrm{f}$. zu den religionsgeschichtlichen Kenntnissen und Hintergründen des Augustinus und ebenso auch Wissowa (1902), 206-216. 
nichts nützen. Sucht sie sich aber ihre Verehrer aus, um ihnen Vorteil zu gewähren, so ist sie nicht zufälliges Geschick. ${ }^{36}$

Ohne viele Worte ist deutlich, dass hier nicht mehr der von Augustinus in den Frühdialogen vertretene positive Begriff von fortuna als einem divinum auxilium zur Erlangung des Besitzes der Weisheit für den Weisen der Polemik zugrunde liegt, sondern im Gegenteil die konventionelle negative Vorstellung von der fortuna als einer unberechenbaren $\tau$ v́ $\eta$, die im Frühdialog Contra Academicos gerade nicht Augustinus, sondern sein Freund Alyphius vertritt. Leider hat Jan den Boeft in seinem eingangs zitierten Artikel über fortuna im Augustinus-Lexikon diese deutliche Akzentverschiebung nicht explizit gemacht, sondern in einer schlichten Aufzählung der Passagen versteckt. Natürlich ist es nicht vollkommen überraschend, dass eine radikale Neuformulierung seiner Theorie über die Kontingenzbewältigung durch Augustinus auch Auswirkungen auf die Konzepte von Schicksal wie Zufall hatte, aber die Deutlichkeit, in der man diese Reformulierung im Blick auf den Begriff fortuna beobachten kann, überrascht dann doch. Sie zeigt sich übrigens auch in der Kritik, die Augustinus in den erwähnten Passagen seiner Retractationes 426/427 n. Chr. an den vierzig Jahre zuvor abgefassten Texten in Contra Academicos übt. Dort kritisiert er sich dafür, die religiöse pagane Dimension des Begriffs fortuna nicht beachtet zu haben und kritisiert es, wenn Menschen der schlechten Gewohnheit folgen, zu sagen: hoc uoluit fortuna, »so will es das Schicksal«, anstatt doch zu formulieren: hoc deus uoluit, »so will es Gott«. ${ }^{37}$ Und nun interpretiert er natürlich auch, wie wir bereits andeuteten, den Hafen der Weisheit, den der Weise nach einstiger Ansicht auf Grund des providentiellen Schicksals, der fortuna als divinum auxilium, erreicht, als die visio beatifica der Seligen. »Unsere Verdienste« und »unsere Natur« sind nun nicht mehr zwei alternati-

36 Augustinus, Civ., IV 18 (CChr.SL 47, 112,9-113,36 Dombart/Kalb): Quo modo ergo dea Fortuna aliquando bona est, aliquando mala? An forte quando mala est, dea non est, sed in malignum daemonem repente conuertitur? Quot sunt ergo deae istae? Profecto quotquot homines fortunati, hoc est bonae fortunae. Nam cum sint et alii plurimi simul, hoc est uno tempore, malae fortunae, numquid, si ipsa esset, simul et bona esset et mala; his aliud, illis aliud? An illa, quae dea est, semper est bona? Ipsa est ergo Felicitas: cur adhibentur diuersa nomina? Sed hoc ferendum est; solet enim et una res duobus nominibus appellari. Quid diuersae aedes, diuersae arae, diuersa sacra? Est causa, inquiunt, quia felicitas illa est, quam boni habent praecedentibus meritis; fortuna uero, quae dicitur bona, sine ullo examine meritorum fortuito accidit hominibus et bonis et malis, unde etiam Fortuna nominatur. Quo modo ergo bona est, quae sine ullo iudicio uenit et ad bonos et ad malos? Ut quid autem colitur, quae ita caeca est passim in quoslibet incurrens, ut suos cultores plerumque praetereat et suis contemptoribus haereat? Aut si aliquid proficient cultores eius, ut ab illa uideantur et amentur, iam merita sequitur, non fortuito uenit. Ubi est definitio illa Fortunae? Ubi est quod a fortuitis etiam nomen accepit? Nihil enim prodest eam colere, si fortuna est. Si autem suos cultores discernit, ut prosit, fortuna non est. An et ipsam, quo uoluerit, Iuppiter mittit? Colatur ergo ipse solus; non enim potest ei iubenti et eam quo uoluerit mittenti Fortuna resistere. Aut certe istam mali colant, qui nolunt habere merita, quibus dea possit Felicitas inuitari. 
ve philosophische Positionen der Neuplatoniker und Ciceros, die vierzig Jahre zuvor erklären, warum wir noch fern der Heimat sind - nein, sie bezeichnen sachidentisch den Grund unserer prinzipiellen Verworfenheit, die Ursünde Adams oder, mit der klassischen Terminologie gesprochen, die Erbsünde. Aus dieser Erbsünde rettet uns kein providentielles Schicksal namens fortuna, wir tragen sie an unserem Leib auch nicht per Zufall umher, sondern wir verdienen sie und einige von uns werden allein aus Gnaden daraus erlöst werden.

Es wäre spannend, diese Entwicklung nun auch noch an einigen weiteren, vor allem antipelagianischen und antidonatistischen Texten des Augustinus $\mathrm{zu}$ verfolgen - das würde unser Bild nicht wesentlich verändern. Mindestens ebenso spannend wäre es daher, nun auch beispielsweise den Umgang des Ori-

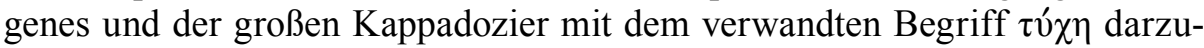
stellen und von dem des Augustinus abzusetzen (schon allein, um zu sehen, ob die duale Modellierung durch Kurt Flasch auch hier am Kern der Sache vorbeiführt). Aber für diese große Aufgabe bedürfte es einer eigenen Studie. Und daher können wir nun diese primär auf Augustinus konzentrierten Ausführungen auch schließen.

\section{Literaturverzeichnis}

Quellen

Aurelius Augustinus, Confessiones, hg. v. Lucas Verheijen, Turnhout 1983 (Corpus Christianorum Series Latina, 27).

Aurelius Augustinus, Contra Academicos. De beata vita. De ordine. De magistro. De libero arbitrio, hg. v. W. M. Green, Turnhout 1970 (Corpus Christianorum Series Latina, 29).

Aurelius Augustinus, De civitate Dei, hg. v. Bernhard Dombart/Alfons Kalb, Turnhout 1955 (Corpus Christianorum Series Latina, 47).

Aurelius Augustinus, De diversis quaestionibus octoginta tribus, hg. v. Bettina Mutzenbecher, Turnhout 1975 (Corpus Christianorum Series Latina, 44A).

Aurelius Augustinus, Dialoge vor den Toren der Stadt. Gegen die Akademiker. Über das Glück. Über die Ordnung, 2. Auflage Zürich 1987.

Aurelius Augustinus, Expositio quarundum propositionum ex epistula apostolica ad Romanos, hg. von Johannes Divjak, Wien 1971 (Corpus scriptorum ecclesiasticorum Latinorum, 84).

Aurelius Augustinus, Retractationum, hg. von Bettina Mutzenbecher, Turnhout 1984 (Corpus Christianorum Series Latina, 57).

Stoicorum veterum fragmenta, Vol. 1: Zeno et Zenonis discipuli, hg. v. Hans von Arnim, Stuttgart 1964.

Stoicorum veterum fragmenta, Vol. 3: Chrysippi fragmenta moralia. Fragmenta successorum Chrysippi, hg. v. Hans von Arnim, Stuttgart 1964. 


\section{Sekundärliteratur}

Bardy, Gustave, La Cité de Dieu, Livres I-V, Paris 1959 (Euvres de Saint Augustin, 33).

Freiherr von Campenhausen, Hans, Lateinische Kirchenväter, 7. Auflage Stuttgart et al. 1995 (Urban Bücher, 50).

Den Boeft, Jan, Art. »Fortuna«, in: Augustinus-Lexikon III, hg. von Cornelius Mayer, Basel 2004, 56-58.

Flasch, Kurt, »Die alte Kirche als Geschichtspotenz Europas«, in: Die Anfänge des Christentums, hg. von Friedich Wilhelm Graf/Klaus Wiegandt, Frankfurt am Main 2009 (Fischer-Taschenbuch, 18277).

Flasch, Kurt, Logik des Schreckens. Augustinus von Hippo, De diversis quaestionibus ad Simplicianum I 2, hg. und erklärt von Kurt Flasch, deutsche Erstübersetzung von W. Schäfer, 2., erweiterte Auflage mit Nachwort, Mainz 1995 (Excerpta classica, 8).

Frederiksen, Paula, »Die frühe Paulusexegese«, in: Augustin-Handbuch, hg. von Volker Henning Drecoll, Tübingen 2007.

Fuhrer, Therese, Augustin, Contra Academicos (Vel de Academicis) Bücher 2 und 3, Einleitung und Kommentar, Berlin/New York 1997 (Patristische Texte und Studien, 46).

Kranz, Margarita, Art. »Zufall«, in: HWdPh XII, hg. v. Joachim Ritter/Karlfried Gründer/ Gottfried Gabriel/Rudolf Eisler/Günther Bien, Basel 2004, 1408-1412.

Lenfant, David, Concordantiae Augustinianae sive Collectio omnium Sententiarum quae sparsim reperiuntur in omnibus S. Augustini operibus, Paris 1656, ND Brüssel 1982.

Lübbe, Hermann, »Kontingenzerfahrung und Kontingenzbewältigung «, in: Kontingenz, hg. v. G. von Graevenitz/O. Marquard, München 1998 (Poetik und Hermeneutik, 17), $35-47$.

Luhmann, Niklas, Soziale Systeme. Grundriß einer allgemeinen Theorie, 14. Auflage Frankfurt am Main 2010 (Suhrkamp-Taschenbuch Wissenschaft, 666).

Markschies, Christoph, »Extra ecclesiam nulla salus? Oder: Wer wird gerettet?«, in: Heil für alle? Ökumenische Reflexionen, hg. v. V. Leppin/D. Sattler, Freiburg im Breisgau 2012 (Dialog der Kirchen, 15).

Schlapbach, Karin, Augustin Contra Academicos (Vel de Academicis) Buch 1, Einleitung und Kommentar, Berlin/New York 2003 (Patristische Texte und Studien, 58).

Wissowa, Georg, Religion und Kultus der Römer, München 1902 (Handbuch der klassischen Altertumswissenschaft, V/4). 
